\title{
CLINICAL AND RADIODENSITOMETRIC EVALUATION OF LOW ENERGY LASER IN MANAGEMENT OF PERIODONTAL CONDITION IN SMOKER PATIENTS
}

\author{
Hisham Mohamed Mahmoud Ali El Sheikh ${ }^{1}$, Ahmed El- Rawdy ${ }^{2}$, Mouchira Salah El Din Mostafa ${ }^{3}$, \\ Wael Selim Amer ${ }^{4}$
}

DOI: $10.21608 / d s u .2020 .21528 .1018$

Manuscript ID: DSU-1912-1018

\section{KEYWORDS}

Low Energy Laser,

Periodontal condition,

Smoker Patients

- E-mail address: hisham.elsheikh@bue.edu.eg

1. Assistant lecturer of Oral Radiology, Faculty of Dentistry, The British University in Egypt

2. Assistant Professor of Oral Radiology, Faculty of Dentistry, Suez Canal University

3. Professor of Oral Medicine and Periodontology and Oral Radiology, Faculty of Dentistry, Cairo University

4. Professor of Oral Radiology, Faculty of Dentistry, Suez Canal University

\section{ABSTRACT}

Aim: This investigation was performed to evaluate clinically and radiodensitometrically the role of Low Energy Laser in the management of periodontal condition in Smoker Patients. Patients and Methods: The study was performed on twentyfour medically free patients having chronic periodontitis. Radiographic bone density evaluation and periodontal parameters were recorded after the first phase of plaque control then; the patients were instructed to perform oral hygiene measures. Patients were distributed into 2 main groups, the first group received Laser therapy, while the second group served as control group. At baseline, three and six months, bone density and alveolar bone level were radiographically recorded using CBCT. Results: The statistical analysis of the collected clinical and radiographic data revealed an overall clinical as well as Radiometric and Densitometric measurements improvement by time for laser group in comparison to control group at baseline, three and six months. Conclusions: The use of Low Energy Laser can enhance bone level and density as well as tissue healing in periodontally affected teeth. In addition, the use of CBCT is considered as an effective method for evaluation of preoperative and postoperative alveolar bone.

\section{INTRODUCTION}

The supportive tissues surrounding the teeth are termed the periodontium and consists of the root cementum, periodontal ligament, alveolar bone and gingival tissue. The composition of the periodontium is affected in many diseases; the most significant is periodontal disease. (1) Periodontal diseases affect the supporting structures of the dentition. Periodontitis been defined as an infection that destruct the alveolar bone and soft tissue of the tooth, which results in pocket formation, gingival bleeding, alveolar bone destruction and finally teeth loss. ${ }^{(2)}$

A large study of risk factors for periodontal disease was undertaken in Erie County, New York State. It showed that those who did not smoke, were at lesser risk for experiencing severe bone loss than those who smoke, with ratios ranging from 3.25 to 7.28 for light and heavy smokers, respectively. ${ }^{(3)}$ 
Salah EI Din and Abdalla studied low energy laser effect on the periodontal structures of abutments supporting over dentures. They concluded that, the mean bone height as well as the mean bone density measurement around the abutments was significantly higher in the laser side than the un lased side. ${ }^{(4)}$

The periodontal therapy success depends on many factors. One of them is the accurate viewing of the morphology of periodontal bone destruction to establish the treatment plan. Radiographs are therefore are very important to determine the severity and extent of the periodontal lesions. ${ }^{(5)}$

Upon comparing clinical probing, 2D radiography and 3D CBCT imaging, CBCT was found to be more effective than others in assessing periodontal structures. CBCT is as accurate as using a periodontal probe in direct measurements and radiographs for interproximal areas. ${ }^{(6)}$

This study was performed to evaluate clinically and radiographically the role of low energy laser in the management of periodontal condition in smoking patients.

\section{PATIENTS AND METHODS}

\section{I-Patient selection:}

The present study was designed as a controlled clinical trial. Patients in this study were recruited from the outpatient clinic of the Oral Periodontology Department, Faculty of Dentistry, Suez Canal University. The number of the study population consisted of 24 young adult smoker patients (as statistically calculated).

\section{Inclusion Criteria:}

Patients included in the present study had to fulfill the following inclusion criteria:
- Adult young male patients

- Smoking patients who consumed 1 pack or more cigarettes per day for at least the past two 2 years.

- Systemically healthy patients with no systemic disease affecting the periodontal status.

- Received no antibiotic therapy for the last 8 months.

- The patients are complaining of bilateral posterior mandibular periodontitis.

\section{II-Patient Grouping:}

The patients were randomly divided into two groups (Group 1 and Group 2).

- Group (1): Compromised 12 patients where Low intensity laser (LIL) was applied to first molar area of the mandible.

- Group (2): Compromised 12 patients as a control group.

\section{III- Pre-treatment Patient evaluation:}

Clinical and radiographic evaluation were carried out for all patients.

\section{Clinical Evaluation:}

For every patient, a detailed history was taken; including present and past medical and dental status. The periodontal status of all cases was assessed according to the following parameters:

A- Plaque index: According to (Silness and loe 1964) ${ }^{(7)}$ the criteria for the plaque index system were:

- 0 . No plaque.

- 1. Film of plaque adherent to gingival margin and the adjacent area of tooth.

- 2. Moderate accumulation of soft deposits within the gingival pocket, on the gingival margin that can be seen by the naked eye. 
- 3. Abundance of soft matter within gingival pocket, gingival margin and adjacent tooth surface.

B- Gingival index: According to (Silness and loe 1964) ${ }^{(7)}$ the criteria for the gingival index system was:

- 0. Normal

- 1. Mild inflammation: slight edema, slight change in color, and no bleeding with probing.

- 2. Moderate inflammation: edema, redness, and bleeding on probing.

- 3. Sever inflammation: extreme redness and edema, ulceration and high bleeding tendency.

C- Probing pocket depth: Measured from the free gingival margin to the base of the pocket depth using William graduated probe.

D- Attachment loss: Measured by subtraction of the distance between gingival margin and cementoenamel junction (GM-CEJ) from recorded probing depth, all measurements were carried out using William's graduated probe.

\section{Radiographic Evaluation:}

\section{CBCT Examination:}

Each case under investigation was scanned radiographically using CBCT (Scanora 3DX ${ }^{* *}$ ) machine with CMOS flat panel detector and isotropic voxel size of $133 \mu \mathrm{m}$ using field of view $(8 \times 10 \mathrm{~cm})$ and high definition mode with exposure parameters of ; $90 \mathrm{Kvp}, 10 \mathrm{~mA}$,exposure time 10s, effective exposure time $6 \mathrm{~s}$, and $0.5 \mathrm{~mm}$ focal spot.

The patients were instructed not to move during exposure. The primary reconstruction time was about 2 minutes which was automatically carried out after acquisition. Images were acquired and saved as DICOM format. Secondary reconstruction was conducted using OnDemand3D software.

\section{IV- Treatment phase:}

\section{»Base line treatment:}

- All patients have done scaling and root planning treatment

\section{Low intensity Laser (LIL) application:}

- Patients in group (1) received (LIL) as follows:

- Application of LIL was performed according to

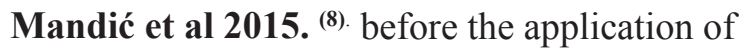
Diode Laser patients were instructed about the potential biological effect of it.

- The low intensity laser was applied in four sessions. The first two sessions were at the same day after periodontal therapy (immediately and after 30-45 minutes). The third and the fourth sessions were carried out after 4 days with an interval of 30-45 minutes between each other .The duration of each of these sessions was 5 minutes using a wave length $904 \mathrm{~nm}$, a frequency of $3000 \mathrm{~Hz}$ (Hertz) and energy output 2 watt.

- During the pre-adjusted time (five minutes), the buccal, the lingual as well as the crestal surfaces were allowed to receive the laser beam. By slow circular motion the applicator tip was moved in a continuous motion to assure the exposure to the laser beam ${ }^{(8)}$.

\section{V- Patient follow-up and post-treatment evaluation:}

- Patients were clinically and radiographically followed up after 3 and 6 months, where all clinical parameters and radiographic linear (radiometric) and densitometric measurements were recorded and used for treatment evaluation. 


\section{Radiographic measurements}

\section{Radiodensitometric analysis:}

Regarding the bone density, it was assessed using OnDemand software ${ }^{(9)}$ where the mean pixel gray scale values of serial ROIs (region of interest) were analyzed to determine whether changes in radio densities have occurred or not ${ }^{(9)}$.

As an attempt to assess the bone density changes around each surface of the studied teeth (first mandibular molar) a ROI was chosen just tangential to the lamina dura on the mesial, distal, buccal or lingual sides according to the site of the bone loss. This ROI was assessed radiodensitometrically as a rectangular area of fixed site and dimensions (figure 1). The mean of the area measurement was pooled and included into further statistical analysis during each of the follow up periods.

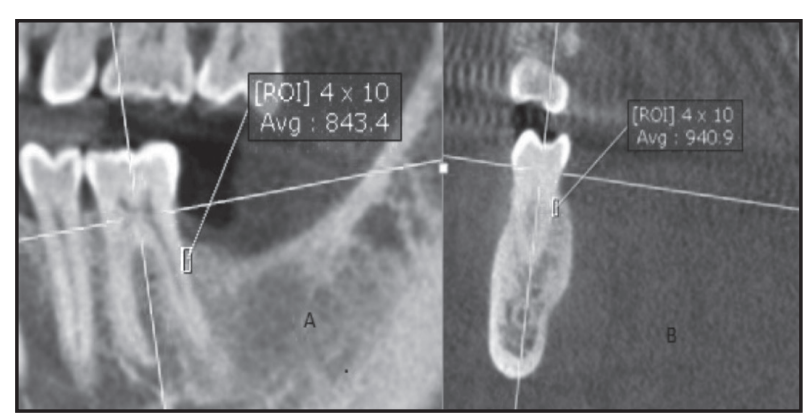

Fig. (1) (A) Sagital Densitometric measurements \& (B) Coronal Densitometric measurements

\section{Radiometric (Linear) analysis:}

Similarly, the marginal bone loss was measured for all patients immediately post-operative, 3 and 6 months, to assess changes in the crestal bone level along the follow up periods in both groups. Measurements of bone level was done in the sagittal and coronal views by drawing a vertical line extending from the CEJ to the crest of the alveolar process. (Figure 2)

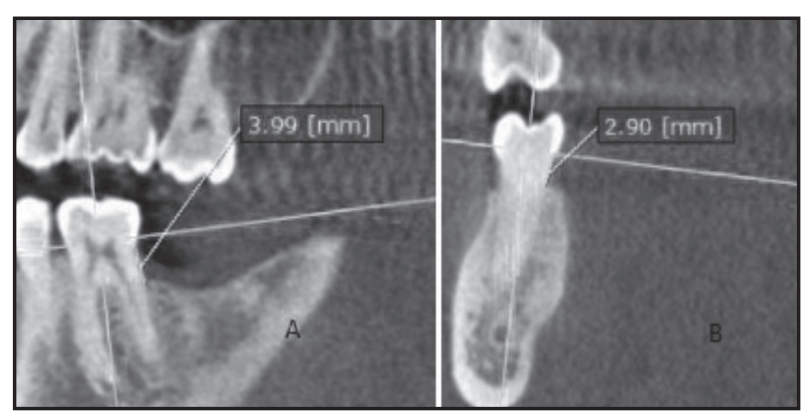

Fig. (2) (A) Sagital linear measurements \& (B) Coronal linear measurements

\section{Statistical analysis:}

For each social event in each test the mean and standard deviation regards were resolved. Data were explored for conventionality using KolmogorovSmirnov and Shapiro-Wilk tests, data showed nonparametric (not-normal) dissemination. To take a gander at between two social occasions in nonrelated models (Groups) Mann Whitney test was used. Friedman test was used to take a gander at between different social affairs in related models (Time periods). The centrality level was set at $\mathrm{P} \leq 0.05$. Truthful assessment was performed with IBM $®$ SPSS $®$ Statistics Version 20 for Windows.

\section{RESULTS}

The obtained data were recorded, tabulated and subjected to statistical analysis.

\section{I) Clinical Results:}

There was no statistically significant difference between the Laser group and the Control group through each follow-up period and the following one. (Baseline), (After 3months) and (After 6 months) regarding the clinical parameters. 


\section{II) Radiographic results:}

\section{1) Densitometric measurements}

Table 1 summarizes the percentage change (increase) in alveolar bone density values through the different follow-up intervals in the laser group and the control group, and compares between both groups at the different follow-up periods. (TABLE 1)

Table (1) Comparison between laser and control groups regarding the percentage of change in density measurements at different follow-up periods:

\begin{tabular}{|c|c|c|c|c|c|c|c|}
\hline \multirow{2}{*}{ Variables } & \multicolumn{6}{|c|}{ Density measurements } \\
\cline { 2 - 7 } & \multicolumn{2}{|c|}{ Pre-3m } & \multicolumn{2}{|c|}{ Pre-6m } & \multicolumn{2}{|c|}{$3 \mathrm{~m}-6 \mathrm{~m}$} & \multirow{2}{*}{ p-value } \\
\cline { 2 - 7 } & Mean & SD & Mean & SD & Mean & SD & \\
\hline Laser & $6.05 \%$ & 4.78 & $11.12 \%$ & 9.72 & $4.65 \%$ & 5.35 & $0.001 *$ \\
\hline Control & $1.00 \%$ & 3.40 & $1.60 \%$ & 4.39 & $0.58 \%$ & 1.92 & $0.700 \mathrm{~ns}$ \\
\hline p-value & $0.008 *$ & $0.006 *$ & $0.034 *$ & \\
\hline
\end{tabular}

*; significant $(p<0.05) \quad n s ;$ non-significant $(p>0.05)$

\section{Laser group:}

There was a statistically significant difference between (Pre-3m), (3m-6m) and (Pre-6m) groups where $(p=0.001)$, in addition, a statistically significant difference was found between (Pre-6m) and each of (Pre-3m) and (3m-6m) groups where $(p=0.002)$ and $(p=0.028)$. However, no statistically significant difference was found between (Pre-3m) and $(3 \mathrm{~m}-6 \mathrm{~m})$ groups where $(p=0.754)$.

\section{Control group:}

There was no statistically significant difference between $($ Pre-3m), $(3 \mathrm{~m}-6 \mathrm{~m})$ and (Pre-6m) groups where $(p=0.0700)$.
Upon comparing both groups, a statistically significant difference was found between them at each follow up interval.

\section{2- Linear Measurements}

Table 2 summarizes the percentage change in linear measurements (decrease) through the different follow-up intervals in the laser group and the control group, and compares between both groups at the different follow-up periods. (TABLE 2)

Table (2): Comparison between laser and control groups regarding the percentage of change in linear measurements at different follow-up periods:

\begin{tabular}{|c|c|c|c|c|c|c|c|}
\hline \multirow{2}{*}{ Variables } & \multicolumn{6}{|c|}{ Linear measurements } \\
\cline { 2 - 7 } & Pre-3m & \multicolumn{2}{|c|}{ Pre-6m } & \multicolumn{2}{|c|}{$3 \mathrm{~m}-6 \mathrm{~m}$} & \multirow{2}{*}{ p-value } \\
\cline { 2 - 7 } & Mean & SD & Mean & SD & Mean & SD & \\
\hline Laser & $6.13 \%$ & 4.37 & $13.50 \%$ & 7.21 & $6.93 \%$ & 5.09 & $<0.001 *$ \\
\hline Control & $0.07 \%$ & 2.04 & $0.78 \%$ & 5.71 & $0.88 \%$ & 5.80 & $0.093 \mathrm{~ns}$ \\
\hline p-value & $0.010 *$ & $0.004 *$ & $0.019 *$ & \\
\hline
\end{tabular}

*; significant $(p<0.05) \quad$ ns; non-significant $(p>0.05)$

\section{a) Laser group:}

There was a statistically significant difference between (Pre-3m), (3m-6m) and (Pre-6m) groups where $(p<0.001)$, in addition, a statistically significant difference was found between (Pre-6m) and each of (Pre-3m) and $(3 \mathrm{~m}-6 \mathrm{~m})$ groups where $(\mathrm{p}=0.002)$ and $(p=0.002)$. However, no statistically significant difference was found between (Pre-3m) and $(3 \mathrm{~m}-6 \mathrm{~m})$ groups where $(p=0.754)$. 


\section{b) Control group:}

There was no statistically significant difference between (Pre-3m), (3m-6m) and (Pre-6m) groups where $(p=0.093)$.

Upon comparing both groups, a statistically significant difference was found between them at each follow up interval.

\section{DISCUSSION}

Over the last 15 years multiple investigations have been performed on the relationship between smoking and periodontal disease, the conclusions of these investigations appear to be strong evidence that smoking confers an increased risk of periodontal disease ${ }^{(\mathbf{1 0})}$.

Tobacco smoking is the main risk factor associated with chronic periodontal disease. The destruction of the supporting tissues of the teeth is considered the typical characteristic of smokingassociated periodontal disease, with the clinical symptoms such as pocket formation, bone loss, attachment loss, and tooth loss ${ }^{(10)}$.

Bragger ${ }^{(11)}$ reviewed the radiographic parameters used for periodontal assessment, their clinical use and biological significance. The review considered digital imaging versus conventional methods, linear measurements from landmarks to alveolar bone crest and tooth and root lengths, furcation radiolucencies and angular defects.

CBCT had been applied in this study following several recommendations in an attempt to minimize the measurement errors, rendering it possible for small bone density changes to be quantitively recorded. The same choice was also previously applied and approved by Eickholz et al ${ }^{(12)}$, since CBCT provides views with shorter examination time and less radiation dose high resolution at lower cost.
Radiographic bone density (relative radiographic grey scale) was assessed as a rectangle covering the investigated area and the changes in density was assessed as comparative values between successive images. The means of the area measurements were pooled as an attempt to eliminate any localization measurement errors ${ }^{(12)}$.

Radio densitometric analysis was performed in this study because it allows detection of density changes between follow up images that relate to change in bone mineral content relative to periodontal disease as proved by Berns et al. ${ }^{(13)}$.

Laser is used to treat various conditions in dentistry. Use of laser needs minimum anesthesia and is time saving for patients. It is the anti-inflammatory, analgesic and bio-stimulative regeneration effects of laser which causes the re-establishment of tissue normal physiologic state. Response to laser therapy also depends on patient tissues and immune system condition $^{(14)}$.

In the present study gallium arsenide laser of wavelength $(904 \mathrm{~nm})$, frequency of $(3000 \mathrm{~Hz})$ and an output power of ( $2 \mathrm{~W})$ in a continuous mode for five minutes, was applied for the patients of the laser group. The $(904 \mathrm{~nm})$ gallium arsenide laser is considered to be the deepest penetration among soft lasers with $5 \mathrm{mms}$ depth penetration. This was in accordance to Khadra et al, as the authors considered that the gallium arsenide laser modulates the activity of cells and tissues surrounding the tooth ${ }^{(15)}$.

Regarding the evaluation of linear bone level there was a statistically significant difference in linear measurements between the laser treated group and the non-laser group through the different follow up periods; baseline, 3 months , 6 months with p value $(0.010,0.004,0.019)$ respectively. These results is in agreement with Zaky et al ${ }^{\left({ }^{(1)}\right)}$ who evaluated the bone healing in maxillary cystic defect reflected by 
digital radiographic assessment of bone density after single post-operative LLLT session and follow up period for 90 days in randomized controlled trial of 16 patients. The results were significant increase in bone length in laser group. This result is in accordance with many previous animal studies that histologically evaluated bone length, density and neo-bone formation following LILT. ${ }^{(16)}$

Regarding the evaluation of relative bone density there was also a statistically significant difference between the laser treated group and non-laser group after 3 months and 6 month of follow up periods. This was within agreement with Kim et al, (17) and Petri et al, ${ }^{(18)}$ who studied the effect of diode laser on human osteoblast grown on titanium implant in animal model. They concluded that LLLT modulated osteoblastic differentiation suggesting possible role in bone formation reflected as an increase in relative bone density or radiographic grey scale.

\section{CONCLUSIONS}

The current study results revealed that the use of LLLT can enhance bone level and density as well as tissue healing in periodontally affected teeth. In addition, the use of CBCT is considered as an effective method for evaluation of preoperative and postoperative alveolar bone.

\section{REFERENCES}

1. Anthony PA. The anatomy and physiology of the healthy periodontium. Gingival Diseases - Their etiology, prevention and treatment. Fotinos Panagakos, $5^{\text {th }}$ ed. 2011; $1-7$.

2. Mailoa J, Lin GH, Khoshkam M, Chan H L and Wang HL. Long -term effect of four surgical periodontal therapies and non- surgical therapy: a systematic review and meta -analysis. J Periodontol. 2015; 86: 1150-1158.

3. Lugo A, La Vecchia C, Boccia S, Murisic B, Gallus S. Patterns of smoking prevalence among the elderly in Europe. Int J Environ Res Public Health. 2013; $10: 4418-4431$.
4. Ebrahimi T, Moslemi N, Rokn AR, Heidari M, Nokhbatolfoghahaie H and Fekrazad R. The influence of low intensity laser therapy on bone healing. J dent. 2012; 9(4):338-348.

5. Braun X, Ritter L, Jervoe-Storm PM and Frentzen M. Diagnostic accuracy of CBCT for periodontal lesions. Clin Oral Invest. 2013;1(4):80-86.

6. Markose E, Vikraman B, Veerabahu M. Three dimensional CT reconstruction: a comparison between 2D, 3D $\mathrm{CT}$ and original anatomical structures. J Maxillofac Oral Surg. 2009; 8(1): 8-12.

7. Silness, J and Loe, H. Periodontal disease in pregnancy II. Correlation between oral hygiene and periodontal condition. Acta Odontol Scand. 1964; 22:121-135.

8. Mandic B, Lazic Z, Markovic A, Mandic B, Mandic M, Dinic A, Milicic B. Influence of postoperative low-level laser therapy on the osseointegration of self-tapping implants in the posterior maxilla: A 6-week split-mouth clinical study. Vojnosanitetski pregled. 2015; 72(3):233-40.

9. Salah El-Din M, Amer W S, El Desouky G G, El Rawdy AM: Radiodentsitrometric evaluation of the effect of low intensity laser therapy on the osseointegration of dental implants in type II diabetic patients. Egyptian Dental journal. 2012; 58: 3081: 3085 .

10. Grossi SG, Nowaldy CA, Takemura A, Ho AW, Genco RJ: Development of an antioxidant supplement for smokers with periodontal disease. J Dent Res 2004: 83: 0192.

11. Bragger U: Radiographic parameters: biological significance and clinical use. Periodontol 2000. 2005; 39:73-90.

12. Eickholz P, Horr T, Klein F, Hassfeld S and Kim TS: Radiographic parameters for prognosis of periodontal healing of infrabony defects: two different definitions of defect depth. J Periodontol. 2004; 75: 399-407.

13. Berns M W, Nelson J S, Wright WH: laser physics and lasertissue interactions. In Achour BM, Vander VM, Berns MW. (eds.): Lasers in plastic surgery and dermatology. 2nd ed., New York, Thieme Medical Publishers, Inc.PP1-10, 1992.

14. Lopes A, Rigau J, Zangaro R, Guidugli-Neto J, Jaeger M. Comparison of the low level laser therapy effects on cultured human gingival fibroblasts proliferation using different irradiance and same influence. Lasers Surg Med. 2010; 29: 179. 
15. Khadraa M, Lyngstadaasb SP, Haanæsa HR, Mustafa K. Effect of laser therapy on attachment, proliferation and differentiation of human osteoblast-like cells cultured on titanium implant material. Biomaterials. 2005; 26: 3503-3509.

16. Zaki BM, Abbas E, Osman N, Elshahat A, Hussein Abdelrazek, El Rafei Shadia. Evaluation of the effect of ozonized water on periodontium of diabetic rats. Der Pharmacia Sinica. 2012; 1:31e40.
17. Kim YD, Kim SS, Hwang DS, Kim SG, Kwon YH, Shin SH, Kim UK, Kim JR, Chung IK. Effect of low-level laser treatment after installation of dental titanium implantimmunohistochemical study of RANKL, RANK, OPG: An experimental study in rats. Lasers in Surgery and Medicine. 2007;39(5):441-450.

18. Petri AD, Teixeira LN, Crippa GE, Beloti MM, de Oliveira PT. Effects of low-level laser therapy on human osteoblastic cells grown on titanium. Brazil Dent J. 2010 ;21(6):491498. DOI: 10.1590/s0103-64402010000600003. 